\title{
Identification of Sunspots on SODISM Full-Disk Solar Images
}

Amro F. Alasta, Abdulrazag Algamudi, RamiQahwaji, Stan Ipson

University of Bradford

United Kingdom

amr_hard@yahoo.com

\begin{abstract}
A new method is proposed to detect sunspots on full-disk solar images recorded by Solar Diameter Imager and Surface Mapper (SODISM) on the PICARD satellite. The procedure of the method is totally automated to detect sunspots and compression with NOAA catalogue shows sunspot recognition rate is $97.8 \%$. The number of sunspots detected by methods shows acquit good agreement with NOAA catalogue Furthermore, calculate the filling factor for W.L. 607nm and compared with SOHO satellite has been presented. The sunspot areas calculated by this method have a high correlation with SOHO in the same period it is $99 \%$.
\end{abstract}

Keywords: Sunspots, SODISM, SOHO, Segmention, PICARD, Wavelength 607 nm and Filling Factors

DOI: $10.6025 / \mathrm{jmpt} / 2018 / 9 / 3 / 102-112$

Received: 4 April 2018, Revised 9 May 2018, Accepted 17 May 2018

(C) 2018 DLINE. All Rights Reserved

\section{Introduction}

There are many satellites observe the solar disk and detect features activates such as Sunspots, PICARD is one of this satellite which launched on 15 June 2010 using SODSIM platform.

Since launch PICARD, SODSIM telescope started recording images 24 hours per 7 days in the year; Level 0 (L0) data which generated every day by PICARD Payload Data Center are registered at BUSOC in Brussels. SODSIM have five wavelengths (centered at 215.0, 393.37, 535.7, 607.1, and 782.2 nm), and provide different images qualities depending on Wavelengths. The combination of solar irradiation and instrumental contamination badly effect on SODSIM and caused degradation because of polymerisation of organic material and, subsequently, irreversible even though the material on the instrument optical surfaces. [1]The channel W.L. $215 \mathrm{~nm}$ lost of the normalized intensity more than 90\% and W.L. 393 about 80\% [2] which shows a pronounced degradation in UV channels as illustrate in figure 1. May be the degradation involved by the polymerisation of contaminants on the front window or on the other optical elements under solar UV exposure while the visible and near infrared channels presents a temporal oscillation but remains relatively constant[1].

102 Journal of Multimedia Processing and Technologies Volume 9 Number 3 September 2018 


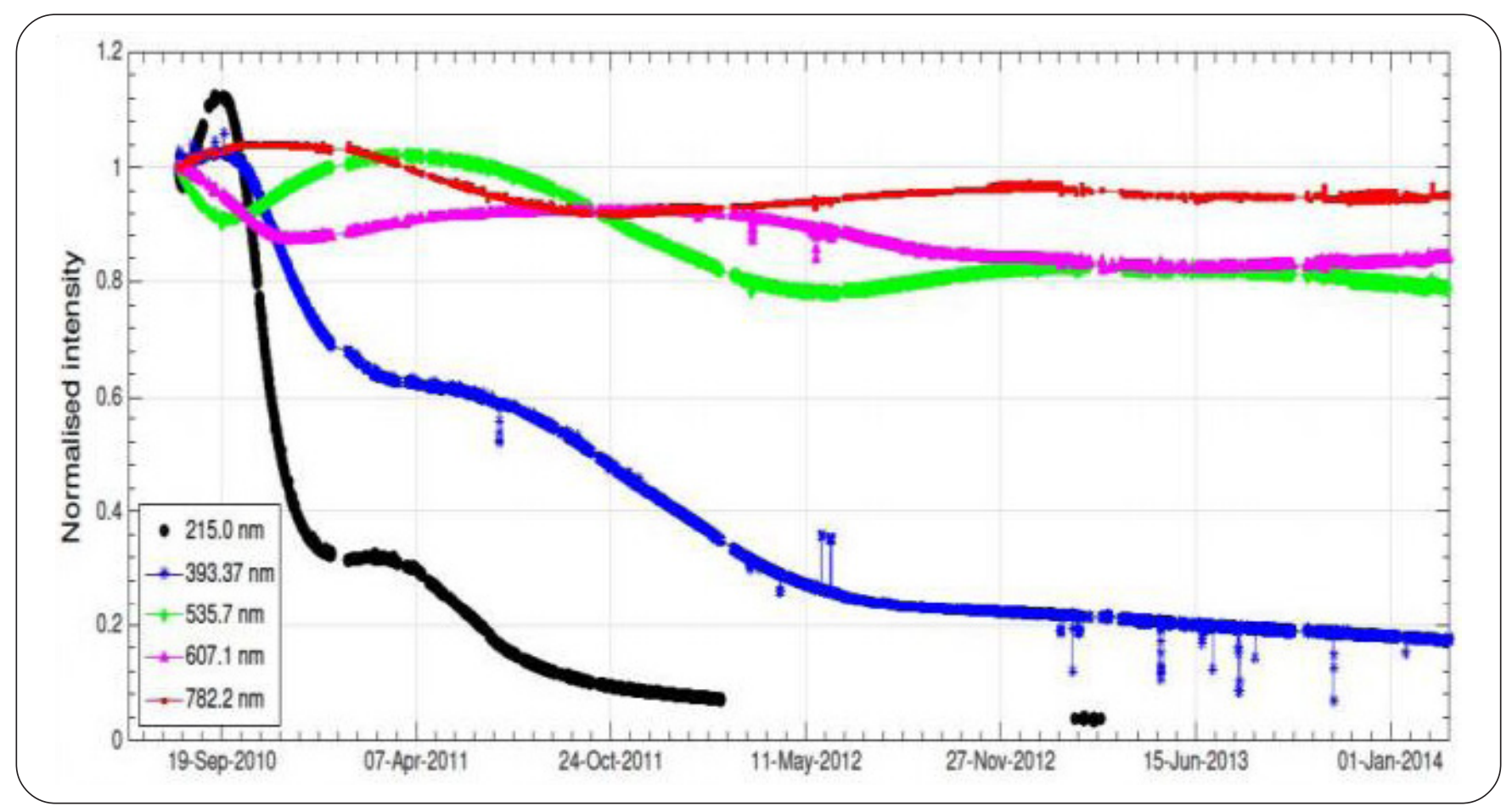

Figure 1. Normalized time series of integrated intensity of PICARD during his mission (Meftah et al. 2015)

According to figure 1, except W.L $215 \mathrm{~nm}$ others wavelengths can be used to detect Sunspots; however, this paper applies only to band $607 \mathrm{~nm}$ images which are available at level 1B1 (level 1B data products include a number of corrections for instrument issues). The total number downloaded was around 250 images. The format of these image files is FITS and each image has a size of $2048 \times 2048$ pixels. The obtained data is from 22th September 2010 to 4th January 2014.

The automated detection of solar features is a technique applied to provide robust, fast and accurate automated detection[3]. There are many researchers which observatories image from satellites such SOHO and SDO and detect features such as sunspots but have been no relatively underused for images from SODSIM, hence, our interest for working with the latter images. Our main contributions in this paper are:

- Provide automated method to detect Sunspots from SODSIM images with verification.

- Provide filling factor a comparison with images from SOHO.

- Provide comparison with manual method.

This paper is organized as follows. Section 2 summarizes some literature survey, Section 3 describes the Pre-processing methods applied, Section 4 shows the accuracy between manual and automated method and Section 5 filling factor computations (calculations of the fractions of solar disk regions occupied by sunspots) presents the some experimental results. Finally conclusions are presented in Section 6.

\section{Literature Survey}

Before segmentation of features on the solar disk can be started it is necessary to determine the boundary of the solar disk (and hence determine its solar radius and center); then we can analyze the interior features. On the solar disk the solar features have a complex distribution of features over widely ranging intensities and with no regular patterns.

\footnotetext{
${ }^{1}$ http://picard.projet.latmos.ipsl.fr/files.php
} 
Sunspots, the features of interest here, are dark, sometimes irregularly shaped, local structures on the solar disk. The automatic sunspots segmentation system outlined here requires no supervision; no previous parameters and no heuristics to perform the stages of segmentation. There are three basic types of segmentation methods:

- Boundary based.

- Region based.

- Thresholding approaches.

Determining the boundaries between regions is done by looking for discontinuities in gray levels as in Zharkov et al. [4] where Gauss smoothing and Sobel gradient are applied to detect contours, using operators sensitive to meaningful discontinuities in intensity level. However, in most applications of this process, especially to complex structures, the results will not be satisfactory, so post-processing operations are required. The review made by Zharkov et al. [5] Evaluated and summarized the existing manual, semi-automated and fully automated feature recognition techniques applied to different solar features including sunspots and networks.

An example of a region based approach is the Watershed method, which produces catchment basins between ridges corresponding to minimum of gradients, often leading to over segmentation. Thus, again, a complex selection of markers and additional modifications are necessary [6].

The simplest and quickest method to apply is thresholding [7]. However, global thresholding of the solar disk is not a practical solution because of the non-uniform brightness of the background solar disk caused by factors such as the limb darkening effect due to varying absorption of light in different thicknesses of the solar atmosphere. However, this can be corrected by normalization of the image brightness in a pre-processing step [8]. Moreover, some background regions of the solar disk in some images could be darker than some sunspots in other regions.

A method for the detection of sunspots from SODISM images (band $535 \mathrm{~nm}$ from 5th August 2010 to 4th January 2014) is introduced in internal work2 [9], Wavelet Harr filter was applied to remove noise from the image, followed by band pass filter to remove limb darkening. Gaussian smoothing was applied next to remove isolated noisy pixels. The correlation coefficient between SOHO and SODSIM images was found to be 0.98 .

In 2008, Curto et al. [7] Developed fully automated recognition method for sunspots (dark features) using morphological operators.

In 2016 M. Meftah and et al. [10] working on SODISM $393 \mathrm{~nm}$ data to detect sunspots and faculae (bright features) applied a similar method to Corto et al. using morphological operations Their method has the following steps:

- Preprocessing steps are applied to SODISM images (L1 data) in order to obtain images with full contrast. Median filtering is applied to reduce noise.

- For sunspot detection, morphological processing consisting of a top-hat operation is applied to the preprocessed images.

- Segmentation of sunspots is performed using an Otsu threshold. However, their method gives better results if manual thresholding is used.

- For faculae detection, morphological processing consisting of a bottom-hat (complement of the top-hat) operation is performed.

- An automated threshold is applied to the image, in order to detect faculae but if the number of detected faculae is not coherent, an iterative procedure is launched, starting from a reduced threshold and/or increasing it gradually until the number of detected faculae belongs to a fixed interval, These steps are the most time consuming.

In 2017 A. Alasta et al.[11] Applied on SODSIM data for W.L. 535nm, their methods summarized in next steps:

${ }^{2}$ https://projects.pmodwrc.ch/solid/index.php/links/10-news-archive/31-deliverables

104 Journal of Multimedia Processing and Technologies Volume 9 Number 3 September 2018 
- Firstly detect the solar disk and record its centre and radius information.

- Secondly convert the image scale from signed 32 bit to unsigned 8 bit,

- Thirdly using Kuwahara and A trous filters to remove noise and some other unwanted features.

- Correct any pixels which are brightness outliers. And apply a Band pass filter to display sunspots on a normalized background.

- Finally Threshold to obtain a mask image determining the sunspot locations.

The results of this method compared with SOHO filling factor and the correlation coefficient between the two data sets is 0.98 .

\section{Pre-processing and Features Detection}

The previews method for detect sunspots have limitation because a manual threshold has been entered. Moreover, these steps are the most time consuming. Also that method applied only on W.L. $393 \mathrm{~nm}$ images and did not applied on huge data.

Our method overcome on problems of time consuming because it is automated and can be applied on massive data for W.L. $607 \mathrm{~nm}$

The method developed to automatically detect sunspots in $607 \mathrm{~nm}$ SODISM L1 images and programmed in MATLAB includes the following steps.
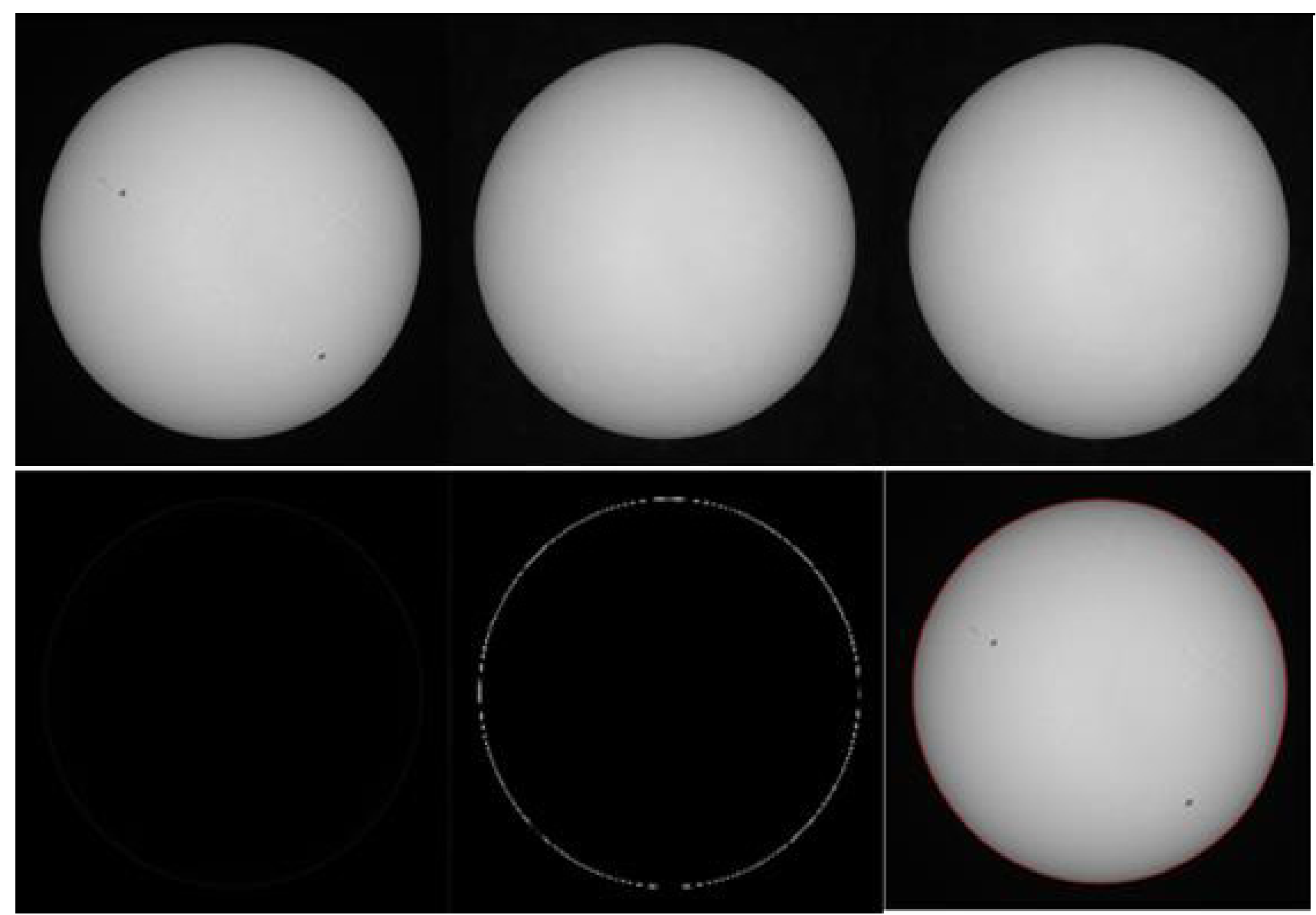

Figure 2. (a) The original image; (b) The filtered image; (c) The image shrunk of the solar disk, the radius is 1 pixel smaller than that in (b); (d) The solar limb shown in grey image; (e) The solar limb shown in the binary image; (f) The solar limb label 


\section{Algorithm 1: Extraction of solar limb}

[i] Get clean solar disk without noise and sunspots, this can be achieved by applying dilation then erosion operation, i.e. a closing operation with a structuring element (SE) on an original SODSIM image

[ii] Choose a circular SE of 30 pixels radius (this is value was chosen by cross validation). Sample result is shown in figure 2.b

[iii] To get the solar limb, the border edges should be determined, the first step shrinks the solar disk by one pixel (filtered image in figure 1.b) to produce a smaller image by 1 pixel

[iv] Then subtracted the new image with filtered image, the result illustrated in figure 2.c

[v] Eliminate CCD noises by utilizing Kuwahara Filter (refer to figure 2.d and 2.e)

[vi] Apply Binary overlay plugin between an original image and solar limb image; it is labeled with a red colour and overlapped on the original image shown in Figure 2.f.

\section{Detection of Sunspots and Verification}

This involves recognising sunspots in solar disk after the solar limb has been extracted. Due to limited resolution of data, the sunspot umbra and penumbra are not separated in SODSIM images; rather they are considered and processed as a whole. The recognition of sunspots is achieved by the steps outlined in Algorithm 2 (refer to Figure 3).

\section{Algorithm 2: Detection of Sunspots}

[i] Original image downloaded from PICARD website is processed using the proposed quality enhancement method; sample output is shown in figure $3 . \mathrm{a}$

[ii] Compute the gradient of the boundaries of sunspots (refer to Figure 3.a)

[iii] Fill holes with a closing operation, this leads to remove dark regions surrounded by bright crests in grayscale images

[iv] Compute the image obtained in 3.a and the gradient image obtained in [ii] (i.e. difference between Figures 3.a and 3.b will yield 3.c)

[v] Separate the gradient of sunspots from noises as shown 1; Figure 3(c), this operation is the threshold on darkness of figure $3(\mathrm{c})$, many experiments have been applied to get suitable value and was $15 \%$ of an intensity of figure 3(c), actually because of

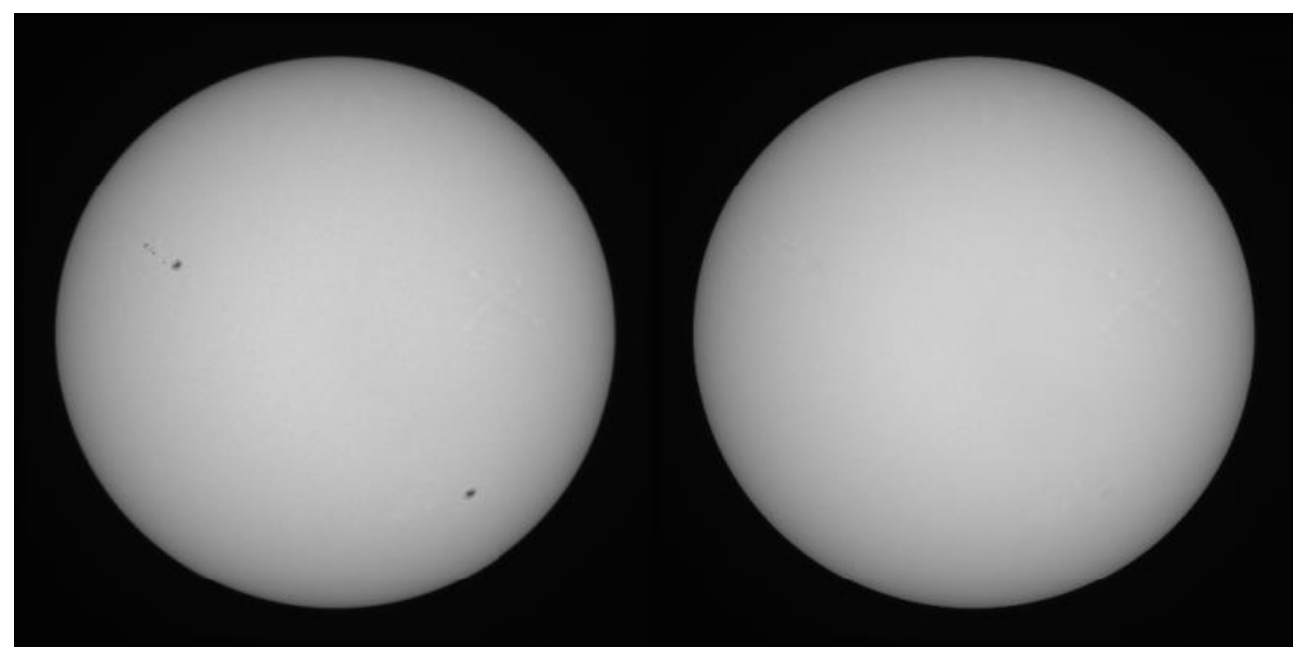

Figure 3: The original image disturbed by instrument noises; (b) the filtered image without sunspots

106 Journal of Multimedia Processing and Technologies Volume 9 Number 3 September 2018


solar limb darkening we note that the sunspots gradient is lower at the solar limb, so the threshold will be $10 \%$ in the region of solar disk.

[vi] Unwanted noises removed by Kuwahara Filter and The Extended Min And Max operation is employed as marker detection for segmentation, figure 3(d) shows sunspot candidate.

[vii] Acquire sunspots from candidates in Figure 3(d); we consider the candidates as verified sunspots in which the difference between the maximum grey value of a pixel and the minimum is bigger than 5 , and other regions are ignored.

[viii] Apply a binary overlay in red color and superimpose the original image, Figure 4 shows sample result.

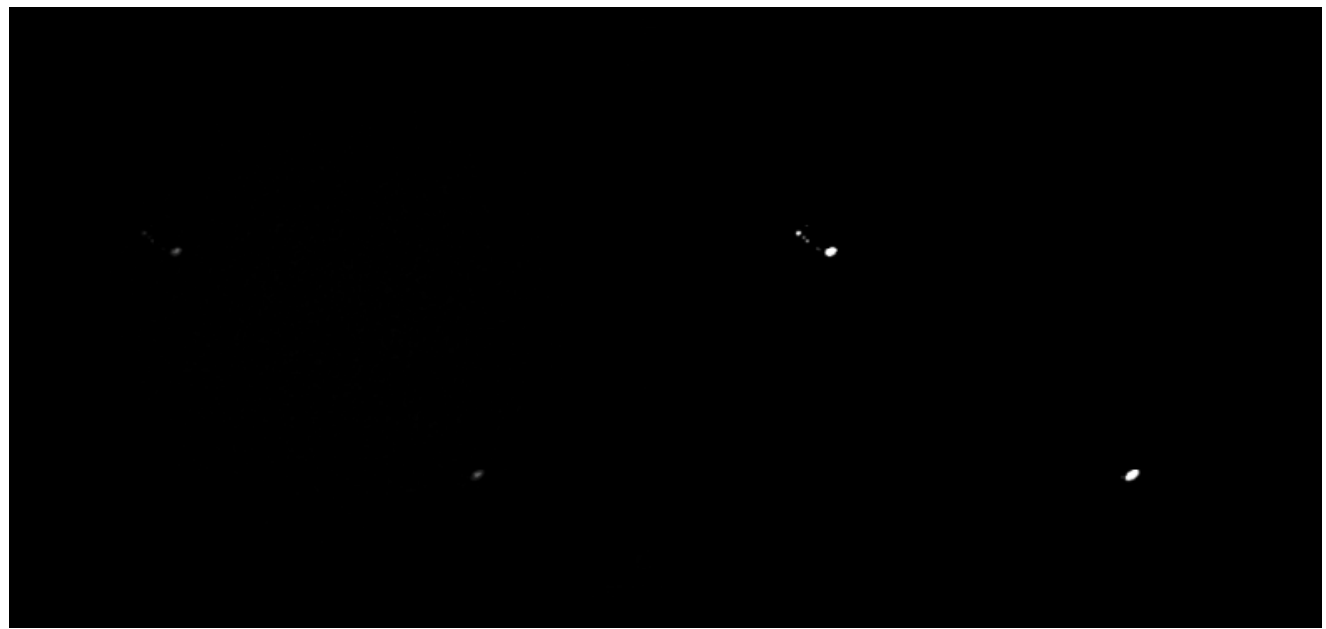

Figure 3c. (c) The gradient on the image; (d) The binary image showing sunspots candidates

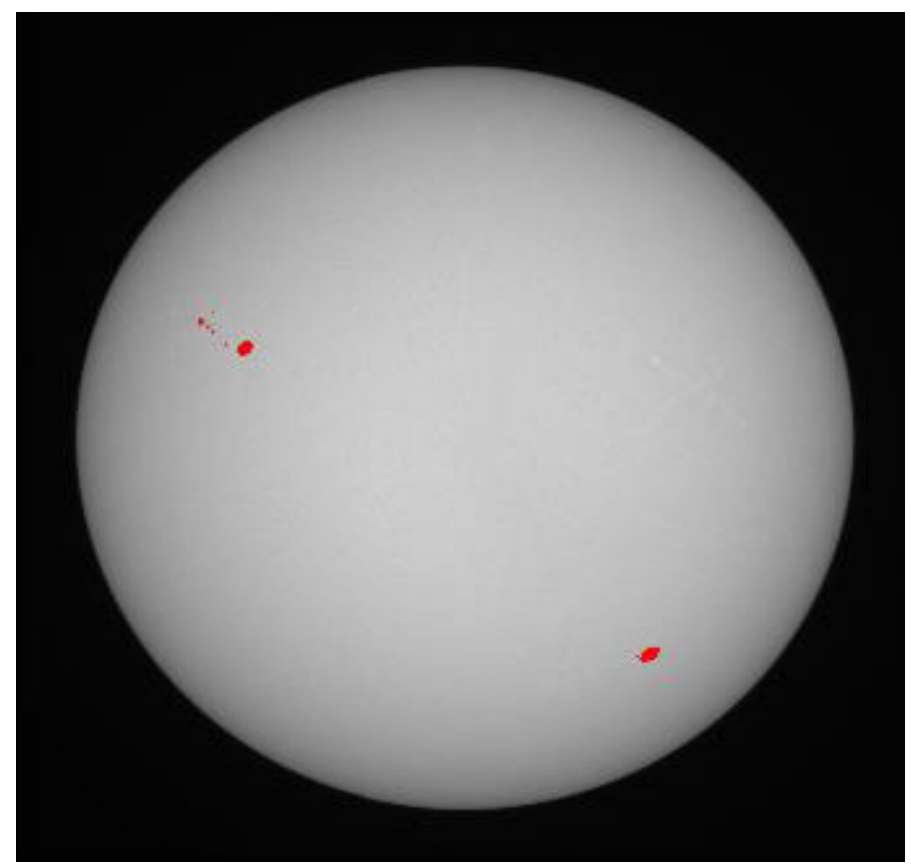

Figure 4. Recognised and superimposed sunspots on the original image 


\section{Accuracy of Automatic Procedure Compared with Manual}

The data of W.L. 607nm from August and September 2010, for both automated and manual methods are calculated and listed in table 1. the table has five columns, first one shows the date on which the image was captured, next to it is the number of sunspots counted by the manual method in an image, the third column shows the number of sunspots detected by our automatic pipeline. The second to the last column shows the false rejection rate (FRR) i.e. the number of sunspots detected by the automatic but not by NOAA catalogue, finally the last column is false acceptance rate (FAR) i.e. the number of sunspots detected by NOAA catalogue but not by the automatic. The rationale for computing FRR and FAR is to evaluate the proposed pipeline in line with what researchers used in the past [4]. SOHO images have been used as reference to overcoming missed small sunspots due manual process because limitation of visible condition. The total of sunspots detected was listed in last row in table for automated and manually respectively. The recognition rate as follows:

$=\frac{\text { Sum of the automatic method }- \text { Sum of the FAR }}{\text { Sum of the NOAA }}$

\begin{tabular}{|c|c|c|c|c|}
\hline Date & Number of spots (automatic method) & Number of spots (NOAA catalogue) & FRR & FAR \\
\hline $5 / 08 / 2010$ & 4 & 4 & 0 & 0 \\
\hline $6 / 08 / 2010$ & 4 & 4 & 0 & 0 \\
\hline $7 / 08 / 2010$ & 4 & 4 & 0 & 0 \\
\hline 9/08/2010 & 4 & 4 & 0 & 0 \\
\hline $10 / 08 / 2010$ & 4 & 3 & 1 & 0 \\
\hline $11 / 08 / 2010$ & 5 & 5 & 0 & 0 \\
\hline $12 / 08 / 2010$ & 4 & 4 & 0 & 0 \\
\hline $13 / 08 / 2010$ & 4 & 2 & 2 & 0 \\
\hline $14 / 08 / 2010$ & 2 & 2 & 0 & 0 \\
\hline $15 / 08 / 2010$ & 2 & 2 & 0 & 0 \\
\hline $16 / 08 / 2010$ & 2 & 3 & 0 & 1 \\
\hline $17 / 08 / 2010$ & 2 & 2 & 0 & 0 \\
\hline $18 / 08 / 2010$ & 2 & 1 & 1 & 0 \\
\hline $19 / 08 / 2010$ & 1 & 1 & 0 & 0 \\
\hline $20 / 08 / 2010$ & 0 & 1 & 0 & 1 \\
\hline $21 / 08 / 2010$ & 0 & 0 & 0 & 0 \\
\hline $22 / 08 / 2010$ & 0 & 0 & 0 & 0 \\
\hline $23 / 08 / 2010$ & 0 & 0 & 0 & 0 \\
\hline $24 / 08 / 2010$ & 0 & 0 & 0 & 0 \\
\hline $25 / 08 / 2010$ & 1 & 1 & 0 & 0 \\
\hline $26 / 08 / 2010$ & 1 & 2 & 0 & 1 \\
\hline $27 / 08 / 2010$ & 1 & 1 & 0 & 0 \\
\hline $28 / 08 / 2010$ & 1 & 1 & 0 & 0 \\
\hline
\end{tabular}

108 Journal of Multimedia Processing and Technologies Volume 9 Number 3 September 2018 


\begin{tabular}{|c|c|c|c|c|}
\hline $29 / 08 / 2010$ & 2 & 2 & 0 & 0 \\
\hline $30 / 08 / 2010$ & 3 & 2 & 1 & 0 \\
\hline $4 / 09 / 2010$ & 4 & 3 & 2 & 1 \\
\hline $5 / 09 / 2010$ & 4 & 4 & 0 & 0 \\
\hline $6 / 09 / 2010$ & 2 & 2 & 0 & 0 \\
\hline 7/09/2010 & 0 & 1 & 0 & 1 \\
\hline $11 / 09 / 2010$ & 0 & 0 & 0 & 0 \\
\hline $12 / 09 / 2010$ & 1 & 2 & 0 & 1 \\
\hline $19 / 09 / 2010$ & 3 & 2 & 1 & 0 \\
\hline 20/09/2010 & 2 & 2 & 0 & 0 \\
\hline $22 / 09 / 2010$ & 2 & 2 & 0 & 0 \\
\hline 23/09/2010 & 2 & 2 & 0 & 0 \\
\hline $24 / 09 / 2010$ & 3 & 2 & 1 & 0 \\
\hline $25 / 09 / 2010$ & 3 & 3 & 0 & 0 \\
\hline $26 / 09 / 2010$ & 3 & 3 & 0 & 0 \\
\hline $27 / 9 / 2010$ & 3 & 3 & 0 & 0 \\
\hline 28/09/2010 & 3 & 3 & 0 & 0 \\
\hline 29/09/2010 & 3 & 3 & 0 & 0 \\
\hline $30 / 09 / 2010$ & 4 & 4 & 0 & 0 \\
\hline Total & 95 & 92 & 8 & 5 \\
\hline
\end{tabular}

Table 1. The comparison of sunspot detected by the automatic procedure with manual one

\section{Filling Factors Computation}

The calculated filling factors for a feature reflect the fraction of the solar disk covered by the feature and assign reference synthetic spectra to it. [12] The filling factor is calculated as a function of radial position on the sun disk. The solar disk is divided by 11 concentric rings starting with an inner radius RI and an outer radius RO. Figure 5 shows filling factor coverage for sunspots.

The data obtained for W.L. $607 \mathrm{~nm}$, was started from 22/09/2010 until 1st January 2014, the filling factor of this sharing data with SOHO have been calculated and comparing with SODSIM in same period time (September, October November and December 2010). The correlation coefficient was $99 \%$ which reflected that methods give excellent results in W.L. 607nm, Figure 6 shows the comparison with $\mathrm{SOHO}$ satellite.

\section{Results}

The segmentation process outlined earlier has been applied to the entire downloaded $607 \mathrm{~nm}$ image data, in order to detect sunspots and calculate their filling factors. Figure 5 shows the results of sunspot filling factor calculations for data from October 2010 until the end of life of the Picard satellite, 1 January 2014. Moreover, in the period from September, 22th 2010 to December 24th 2010 figure 6, when data from both sources are available, a comparison between sunspots' filling factors calculated for 
SODISM $607 \mathrm{~nm}$ images and MDI intensitygram images from the SOHO satellite has been done. The most common of compering method between two values or measure of dependence between two quantities is "Pearson's correlation coefficient", commonly called "the correlation coefficient". This is obtained by dividing the covariance of the two variables by the product of their covariance of the two variables by the product of their standard deviations.

\begin{tabular}{|c|c|c|}
\hline Index & Inner radius (relative radius) & Outer radius (relative radius) \\
\hline 1 & 0.00 & 0.07 \\
\hline 2 & 0.07 & 0.16 \\
\hline 3 & 0.16 & 0.25 \\
\hline 4 & 0.25 & 0.35 \\
\hline 5 & 0.35 & 0.45 \\
\hline 6 & 0.45 & 0.55 \\
\hline 7 & 0.55 & 0.65 \\
\hline 8 & 0.65 & 0.75 \\
\hline 9 & 0.75 & 0.85 \\
\hline 10 & 0.85 & 0.95 \\
\hline 11 & 0.95 & 1.05 \\
\hline
\end{tabular}

Table 2. Relative radius values

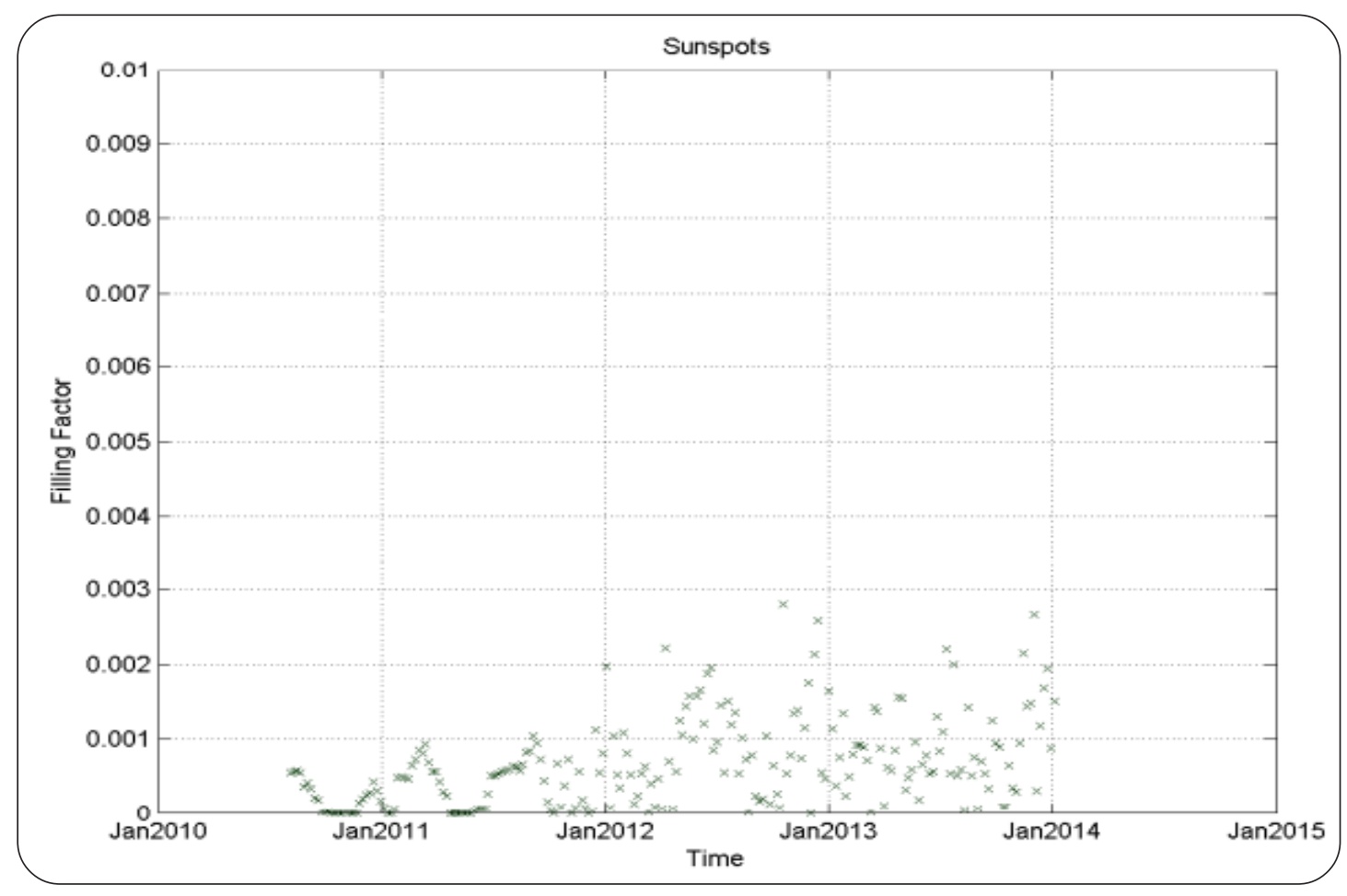

Figure 5. The filling factors (area coverage) for sunspots 


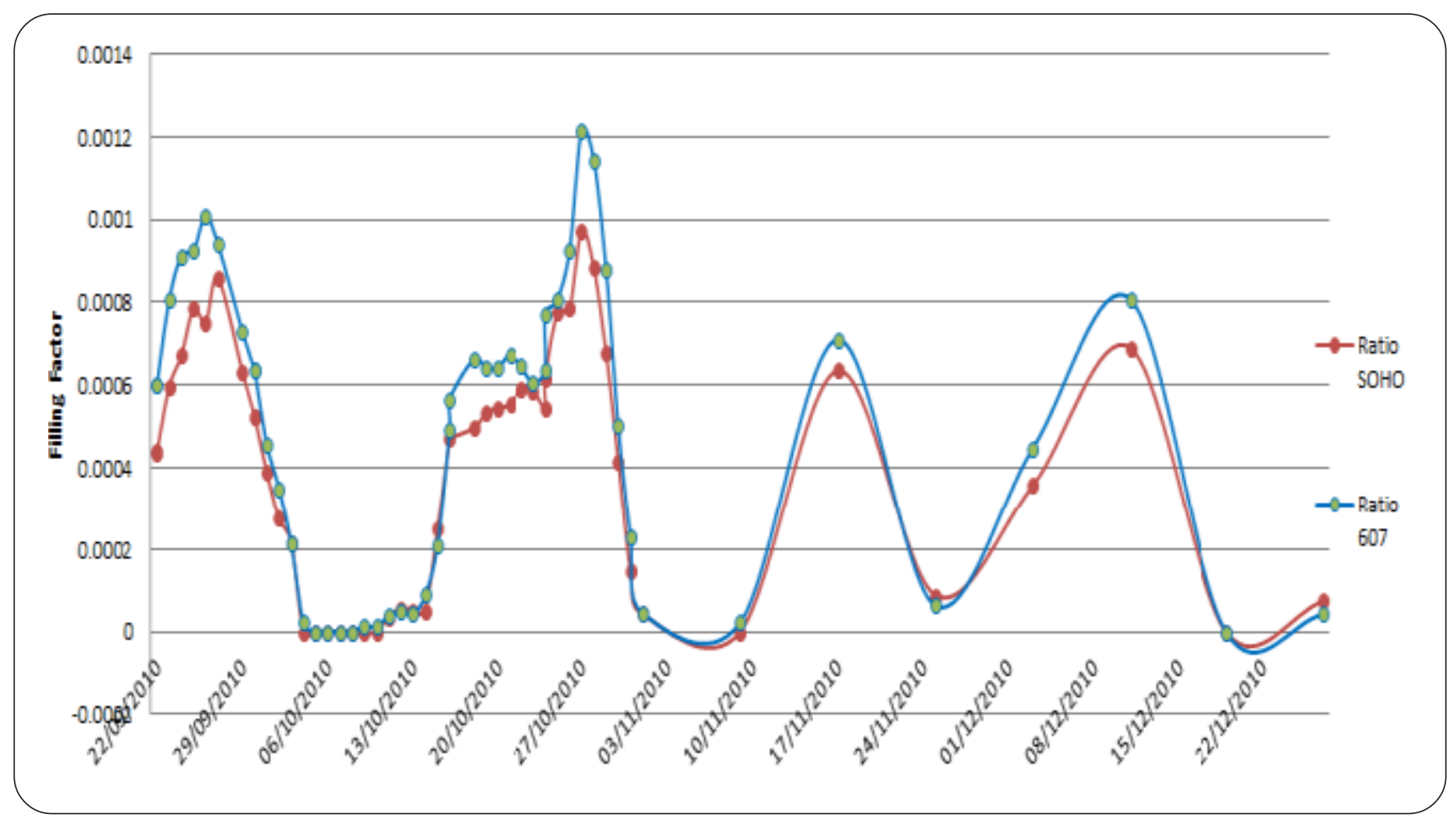

Figure 6. A comparison of sunspot filling factors calculated from SOHO and SODISM images from 22 th September 2010 until 25 December 2010

\section{Conclusions}

Using suitable automated methods for detect sunspot of SODSIM images is possible even though SODSIM image degradation through the lifetime of PICARD The biggest advantage is reduced consuming time, there are few methods applied to segmented SODSIM images the first was by M. Meftah et al, which applied to W.L. $393 \mathrm{~nm}$ but needs to manually interaction to optimize the threshold which is calculated using the Otsu method [7], second method was by Qahwaji et al in his internal work, shows good results and a correlation coefficient of 0.98 [9] between SODISM and SOHO images, only slightly less than that achieved in the same period in third method by Alasta et al, which successfully detects sunspots on W.L. 535 nm images over the lifetime of the satellite and then calculates their filling factors, Furthermore, the comparison of sunspots' filling factors between SOHO and SODSIM images shows an excellent match over the early period when they are both available, with a correlation coefficient of 0.985 [11]. Our method here is applied to W.L. $607 \mathrm{~nm}$ is completely automated which make it easy to apply to massive data images. The correlation coefficient is 0.99 which reflect excellent results the results in Figure 6 shows the filling factors for SODISM and SOHO are systematically slightly different in amplitude despite mostly changing instep. This is most evident over the period from 22 Sep 2010 to 29 Sep 2010 when there is a somewhat lower correlation coefficient $(\approx 0.98)$ and this is still better than from the previous method with W.L. 535 in the same period which shows correlation 0.92. This may well be related to the fact that the SOHO data corresponds to a different wavelength $(676.8 \mathrm{~nm})$ to the SODSIM images. However, this is first automated method achieve 0.99 corrections between SOHO and SODSIM. Regarding to table 2 as a key technique for identifying regions of interest, image segmentation was also explored, investigated and deployed. Similarly, a thorough evaluation and comparison of the results to similar works was done. In general the system developed in this paper has proven to be promising, out of 92 sunspots, it automatically detected around 98\% comparing with NOAA catalogue of the sunspots.

\section{References}

[1] Meftah, M., a Hauchecorne, Corbard, T., Bertran, E., Chaigneau, M., Meissonnier, M. (2014). PICARD SODISM, a space telescope to study the Sun from the middle ultraviolet to the near infrared, no. (January), p. 1-38.

[2] Meftah, M., Irbah, A., Hauchecorne, A., Hochedez, J.-F. (2013). "PICARD payload thermal control system and general impact 
of the space environment on astronomical observations," no. (May), p. 87390B, 2013.

[3] Qahwaji, R., Colak, T. (2005). “Automatic detection and verification of solar features,” Int. J. Imaging Syst. Technol., vol. 15, p. $199-210$

[4] Zharkova, V., Ipson, S., Benkhalil, A., Zharkov, S. (2005). Feature recognition in solar images, Artif. Intell. Rev., 23 (3) $209-266$.

[5] Zharkov, S., Zharkova, V., Ipson, S., Benkhalil, A. (2005). Technique for automated recognition of sunspots on full-disk solar images, EURASIP J. Appl. Signal Processing, 2005 (15) 2573-2584.

[6] Kacprzyk, E. J. (2011). Soft Computing in Industrial Applications, Soft Comput., June. 438.

[7] Curto, J. J., Blanca, M., Martínez, E. (2008). Automatic sunspots detection on full-disk solar images using mathematical morphology, Sol. Phys., 250 (2) 411-429.

[8] Zhang, S., Yang, H., Singh, L. (2014). Increased information leakage from text, In: CEUR Workshop Proc., 1225. $41-42$.

[9] Rami Qahwaji, O. A. (2015). Stan Ipson”SOLID D3.5 Filling factors catalogue for PICARD images SOLID, First Eur. Compr.Sol. Irradiance Data Exploit. p. 1-8, 2015.

[10] Meftah, M., Corbard, T., Hauchecorne, A., Irbah, A. P., Boumier, Chevalier, Schmutz, W., Ikhlef, R., Morand, F., Renaud, C.,Hochedez, J.-F., Cessateur, G., Turck-Chièze, S., Salabert, D., Rouzé, M., van Ruymbeke, M., Zhu, P., Kholikov, S., Koller, S., Conscience, C., Dewitte, S., Damé, L., Djafer, D. (2016). Main results of the PICARD mission, no. (July) p. $99040 Z$.

[11] Alasta, A. F., Algamudi, A., Qahwaji, R., Ipson, S., Nagern, T. A. (2017). Automatic sunspots detection on SODISM solar images, In: 2017 Seventh International Conference on Innovative Computing Technology (INTECH), p. 115-119.

[12] Ashamari, O., Qahwaji, R., Ipson, S., Schöll, M., Nibouche, O., Haberreiter, M. (2015). Identification of photospheric activity features from SOHO/MDI data using the ASAP tool, J. Sp. Weather Sp. Clim., vol. 5, p. A15. 